\title{
Supplementary Materials, for
}

\section{Acoustic Ejection Mass Spectrometry for High-Throughput Analysis}

Hui Zhang ${ }^{1, *}, \dagger$, Chang Liu ${ }^{2, *}{ }^{*} \dagger$, Wenyi Hua ${ }^{1, \dagger}$, Lucien P. Ghislain ${ }^{3, \dagger}$, Jianhua Liu ${ }^{1}$, Lisa Aschenbrenner ${ }^{1}$, Stephen Noell ${ }^{1}$, Kenneth Dirico ${ }^{1}$, Lorraine F. Lanyon ${ }^{1}$, Claire M. Steppan ${ }^{1}$, Don

W. Arnold ${ }^{2}$, , Thomas R. Covey ${ }^{2}$, Sammy S. Datwani ${ }^{3, *}, \S$, Matthew D. Troutman ${ }^{1}$

\section{Affiliations:}

${ }^{1}$ Pfizer Global Research and Development, Eastern Point Road, Groton, CT, 06340 USA.

${ }^{2}$ SCIEX, 71 Four Valley Drive, Concord, ON, L4K 4V8 Canada.

${ }^{3}$ Beckman Coulter Inc., San Jose, CA, 95134 USA.

$\dagger$ These authors contributed equally to this work.

*Correspondence to:

Hui Zhang, Hui.Zhang3@pfizer.com

Chang Liu, $\underline{\text { Chang.Liu@sciex.com }}$

Sammy S. Datwani, SDATWANI@beckman.com

†Current address: Veristad LLC, Livermore, CA, 94550 USA

§Current address: Synthego Corporation, 600 Sagnaw Drive, Redwood City, CA 94063 USA 
This file includes:

- The ADE Technology

- ADE-OPI-MS Analytical Performance

- Applications

- Supplemental Figures and Legends: S1 to S7

- Supplemental Table and Legend: S1, S2

- Supplemental Movie Legend: S1

Other Supplementary Materials for this manuscript include the following:

Separate File for Movie S1 


\section{The ADE Technology}

Acoustic droplet ejection (ADE) technology is optimized to deliver small volumes of solution from source to destination using acoustic energy. Historically, this technology has been integrated with an inverted microplate held on a destination stage for drug discovery applications (compound reformatting, dose response setup) and for screening applications (pharmacology, ADME), proteomics and genomics ${ }^{1}$. At each well of the source microplate, a piezoelectric transducer with an input waveform at $10 \mathrm{MHz}$ center frequency $(\mathrm{CF})$ is used to generate a focused ultrasonic pulse which propagates through the coupling fluid (figure not shown) into the walls and contents (Fig. 1A) ${ }^{2}$. This ultrasonic pulse is reflected at the interfaces (including the fluid meniscus) and returns to the piezoelectric transducer for real-time processing to audit the microplate (e.g. to measure the: fluid meniscus position; the fluid meniscus tilt; the fluid impedance). At greater burst amplitude acoustic energy is focused at the sample fluid surface and acoustic pressure is applied to form a mound at the fluid meniscus. These ultrasonic pulses reflected by the fluid menisci are processed by dynamic fluid analysis (DFA) algorithms to determine droplet ejection parameters.

Next, a burst pulse is then applied to acoustically transfer droplets. Droplet diameter is in the range $120-360 \mu \mathrm{m}(1-25 \mathrm{~nL}$ in volume), with a typical droplet velocity of $1 \mathrm{~m} / \mathrm{s}$ that is directed to the target ${ }^{3}$. The ADE repetition rate of this process is $200-800 \mathrm{~Hz}$, transferring a droplet train from the same sample well at an effective infusion flow rate of $30-75 \mu \mathrm{L} / \mathrm{min}$ (Figure S1A and S1B). The typical injection volume is a single droplet $(2.5 \mathrm{~nL})$, and a droplet train of 10 or more droplets is possible. ADE allows for precise and consistent transfer of a 
selected number of sample droplets into the OPI for analysis as a single peak. The entire system operates at room temperature and pressure. With acoustic fluid calibrations optimized for specific fluid classes defined by surface tension and viscosity, ADE can be used with a variety of solutions with equivalent accuracy and precision (Figure S2).

\section{Materials:}

Sodium hydroxide was purchased from J.T. Baker, 5674-02. Echo® qualified 384-well polypropylene microplates were purchased from Labcyte Inc., P-05525. For droplet volume verification we utilized 384-well clear-bottom polystyrene microplates were purchased from Greiner Bio One, 781096 and a Synergy H4 Hybrid multi-mode microplate reader purchased from BioTek Instruments, Inc. All other chemicals and reagents were purchased from SigmaAldrich.

\section{Method:}

In the droplet volume verification method, each test solution was prepared with $0.15 \mathrm{mM}$ sodium fluorescein as a fluorescence tracer dye. The ADE liquid handler was setup for twenty droplet $(50 \mathrm{~nL})$ transfers from each test solution prepared in an Echo qualified 384-well source microplate into a clear-bottom 384-well destination microplate. The 384-well source plate for each solution was prepared in a quadrant fill pattern, with 96 wells filled to each of four volumes: $15,20,30$, and $65 \mu \mathrm{L}$. Following the transfers, the clear-bottom microplates were backfilled with $50 \mu \mathrm{L}$ per well of $10 \mathrm{mM}$ sodium hydroxide using a conventional bulk filler, centrifuged for $1 \mathrm{~min}$ at $200 \mathrm{RCF}$ and then incubated for $30 \mathrm{~min}$ at room temperature. Next, the microplate was read on the Synergy fluorescence reader to determine the fluorescence level in 
each well. The fluorescence level was compared to a standard curve to determine the transfer volume for each well. Fig. 2 shows the average transfer volume and coefficient of variation (CV) results for the following fluids: glycerol (0-60\%, 10\% steps), dimethyl sulfoxide (DMSO) (70$100 \%, 5 \%$ steps $)$, fetal calf serum (FCS) (0-100\%, 20\% steps). Triton X-100 (0-200\% CMC, 0 , 5, 14, 200\%). DMSO dilutions are in Milli-Q $\mathrm{H}_{2} \mathrm{O}$, the remaining dilutions were prepared with $1 \mathrm{X}$ phosphate buffered saline (PBS). Triton X-100 dilutions are $0.001 \%, 0.003 \%$, and $0.042 \%$ (v/v), these concentrations represent $5 \%, 14 \%$, and $200 \%$ of the critical micelle concentration (CMC), respectively. Fluid calibrations for organics including acetonitrile $\left(0-100 \%\right.$ in $\left.\mathrm{H}_{2} \mathrm{O}\right)$ and methanol (up to $50 \%$ in $\mathrm{H}_{2} \mathrm{O}$ ) are also available (data not shown).

\section{ADE-OPI-MS Analytical Performance}

First, we have explored the potential to increase overall sample analysis speed by deploying multiplex analysis. Figure S3 shows a trace of 384 samples recorded in $<170 \mathrm{sec}(2.2 \mathrm{~Hz})$ by multiplexing four different compounds (omeprazole, quinidine, midazolam, bupropion) ejected sequentially from individual wells. Importantly for this multiplexed approach, the precision remained high with $\mathrm{CV} \%$ between $3 \%$ to $8 \%$ for these model compounds. After obtaining highly accurate and precise data across a wide dynamic range for small molecules and peptides, we investigated larger molecules. In Figure S6, we show the analysis of an antibody standard (MW 150K, Waters, Milford, MA) with alternating five droplet (12.5 nL) injections of the standards at two concentrations, $667 \mathrm{nM}$ and $67 \mathrm{nM}$ in aqueous solution. The estimated LOD is $<1$ fmole loading.

\section{Additional ADE-OPI-MS Pharmacology Application with Methods}




\section{Choline uptake assay:}

Experiment details:

In 384-well poly-D-lysine coated plates, 50,000 cells per well were plated in $50 \mu \mathrm{L}$ media and recovered overnight. The cells were rinsed and equilibrated for $30 \mathrm{~min}$ in $50 \mu \mathrm{L}$ HBSS buffer and then pre-incubated with test compounds for $15 \mathrm{~min}$. A final concentration of $100 \mu \mathrm{M} \mathrm{d} 9$ Choline substrate was added and uptake progressed for $15 \mathrm{~min}$. Substrate was removed by aspiration and the cells were washed two times with HBSS buffer. The wells were then extracted with $30 \mu \mathrm{L}$ of HPLC grade acetonitrile:methanol:water $(2: 2: 1)$.

Lysed cell samples were analyzed using the ADE-OPI-MS platform without further treatment or cleanup and compared to a conventional LC-MS method ${ }^{4}$. Pre-incubation of HC-3 and STS showed well defined inhibition and activation profiles as expected. Both profiles generated with ADE-OPI-MS had high concordance when compared to EC50 generated via conventional LC/MS. The ADE-OPI-MS platform increased the speed 10-fold and reduced sample volume 500-fold while delivering equivalent data quality and concordance for both inhibition and activation profiles of the two modulators. Further, the ADE-OPI-MS platform results were obtained without an internal standard (IS) and with higher precision than the LC-MS methods with IS. 


\section{ADE-OPI-MS experimental conditions:}

OPI carrier solvent flow: methanol $(0.20 \mathrm{~mL} / \mathrm{min})$, sample ejection volume: $5 \mathrm{~nL}$, mass spectrometer: SCIEX Triple Quad ${ }^{\mathrm{TM}}$ 6500+ system. Data collection: Analyst 1.6, MultiQuant 2.1, ion source temperature: $150^{\circ} \mathrm{C}$, analyte SRM Transitions Monitored (CE) D9-Choline $113.2 \rightarrow 69.1(26 \mathrm{eV})$

We also demonstrate a first implementation of the ADE-OPI-MS platform in a high-throughput screening (HTS) drug discovery application (Fig. S7). The choline transporter (CHT) is an attractive target for neurological disorders such as Alzheimer's and attention deficit hyperactivity disorder (ADHD) as it is known to mediate synthesis and distribution of the critical neurotransmitter acetylcholine. Conversely, cholinergic dysfunction is also associated with attention deficiencies and compromised motor neuron function. In order to screen for modulation of CHT activity by a candidate drug molecule, a cellular uptake assay was developed and validated to monitor the uptake of the deuterated (D-9 labelled) choline, as choline has high endogenous background levels in the HEK293 cell line. HEK cells expressing the high affinity CHT were used for this assay. Known modulators hemicholinium-3 (HC-3, inhibitor) and staurosporine (STS, activator) (7) were tested at a range of dose concentrations. Due to high background signal from endogenous choline, D-9 labelled choline was used as substrate. Overall the ADE-OPI-MS platform delivers very robust Z' and consistent EC50s determination for both activation and inhibition when compared with LC-MS approach. 


\section{Supplemental Figures and Legends}

Figure S1: ADE-OPI-MS Breadboard System

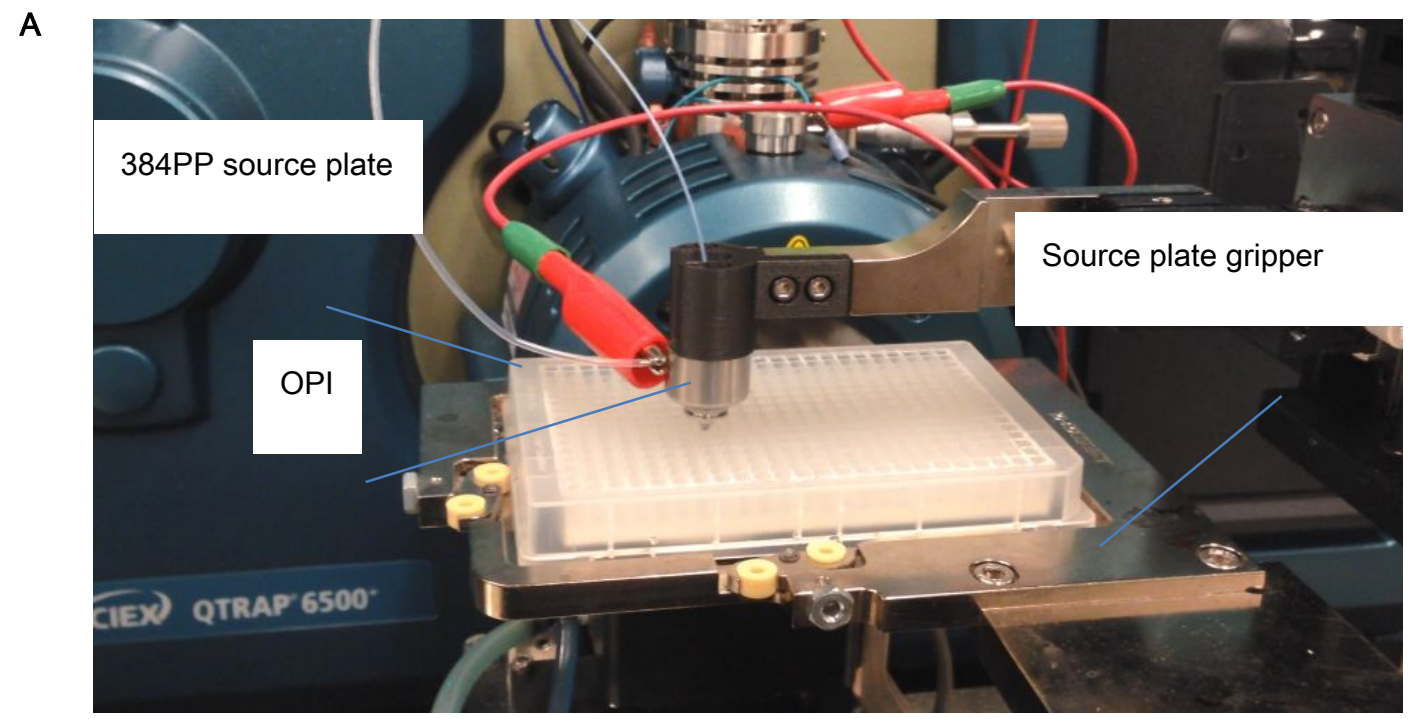

384PP source plate

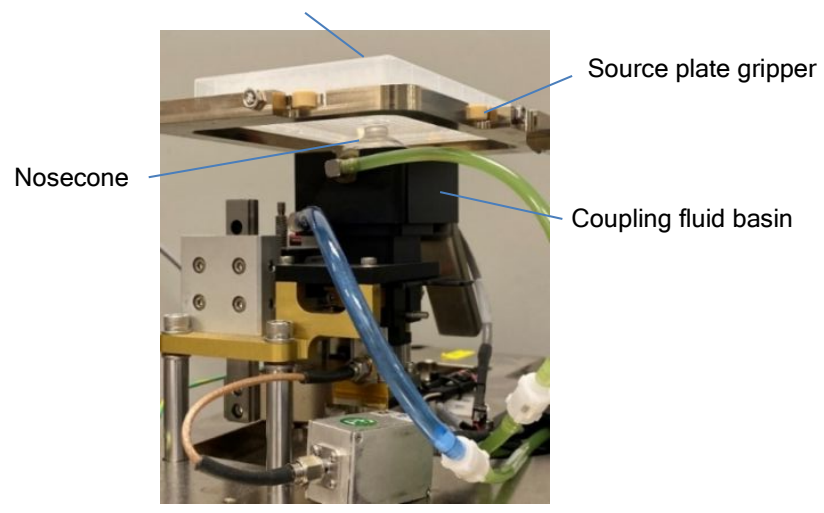

C

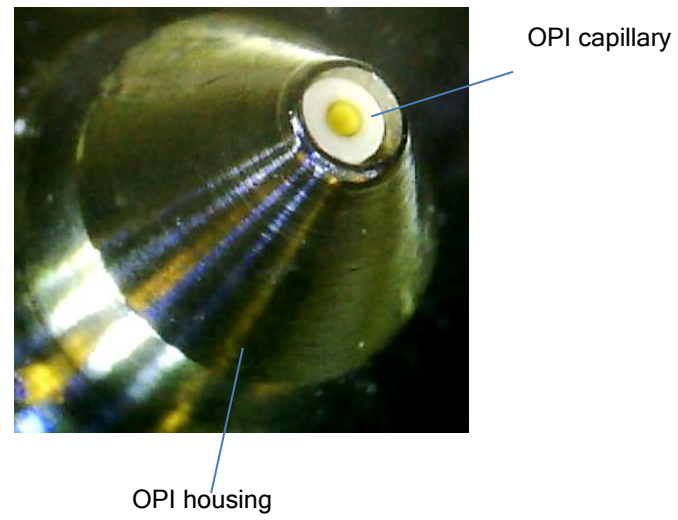




\section{Fig S1 Legend:}

ADE-OPI-MS breadboard system. (A) Photograph of the ADE-OPI-MS platform showcasing a 384 polypropylene source microplate in the source tray of a breadboard XY stage. The system is mechanically aligned with an Echo acoustic transducer (below stage), center of the microplate source well, and the OPI transfer capillary on a common axis. Clearance between microplate and OPI is set to $1-2 \mathrm{~mm}$ to provide for droplet placement to the center of the OPI capture region. A transfer capillary connects the OPI to a SCIEX Triple Quad ${ }^{\mathrm{TM}} 6500+$ MS with IonDrive ${ }^{\mathrm{TM}}$ Turvo V ESI source. (B) Photo of ADE setup showing transducer housing, coupling fluid basin, nosecone, source plate gripper and 384PP source plate. (C) zoom-in view of OPI probe showing ID of stainless-steel outer tube and inner capillary. Photo Credit: Chang Liu, SCIEX, and Lucien P. Ghislain, Beckman Coulter Life Sciences. 
Figure S2: Acoustic Fluid Calibration for ADE Droplet Volume and Coefficient of Variation

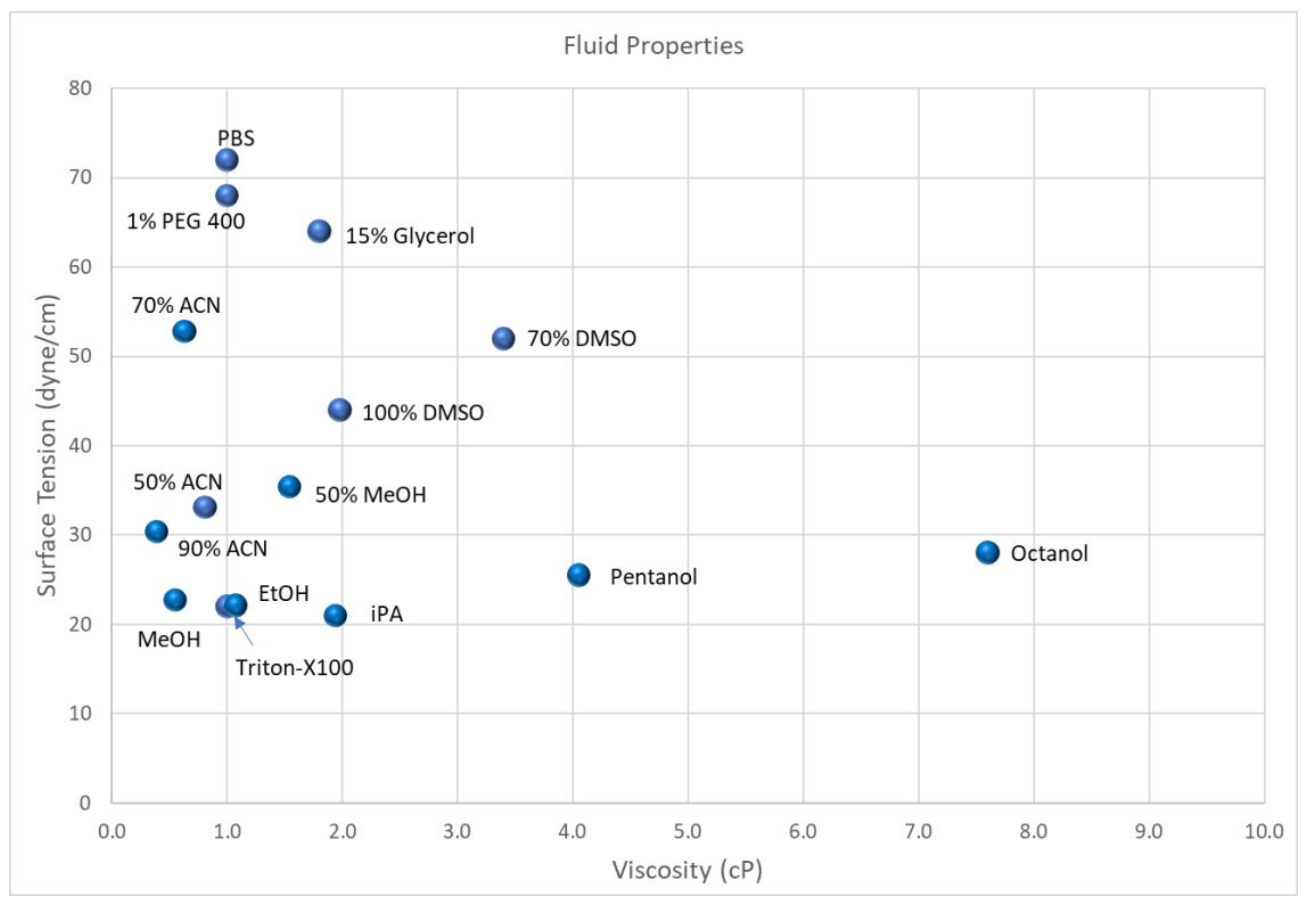

C

Echo Transfer Volume Accuracy

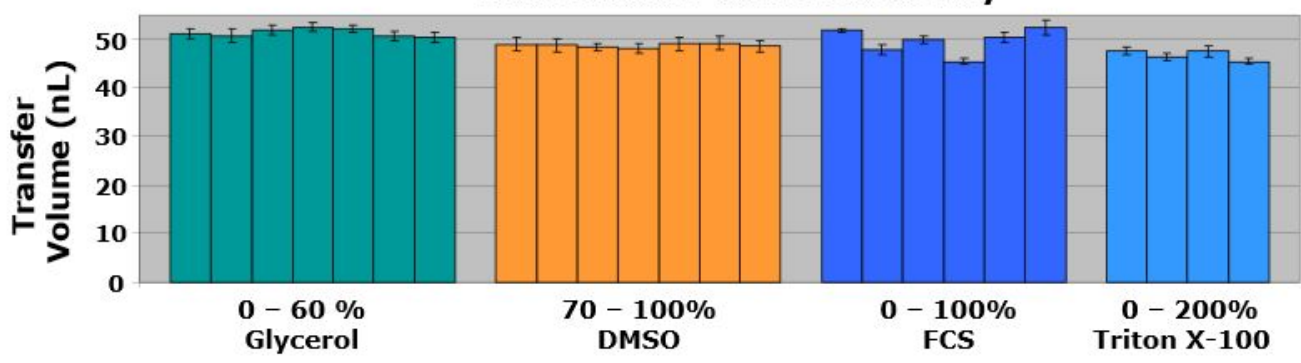

Echo Transfer Volume Precision

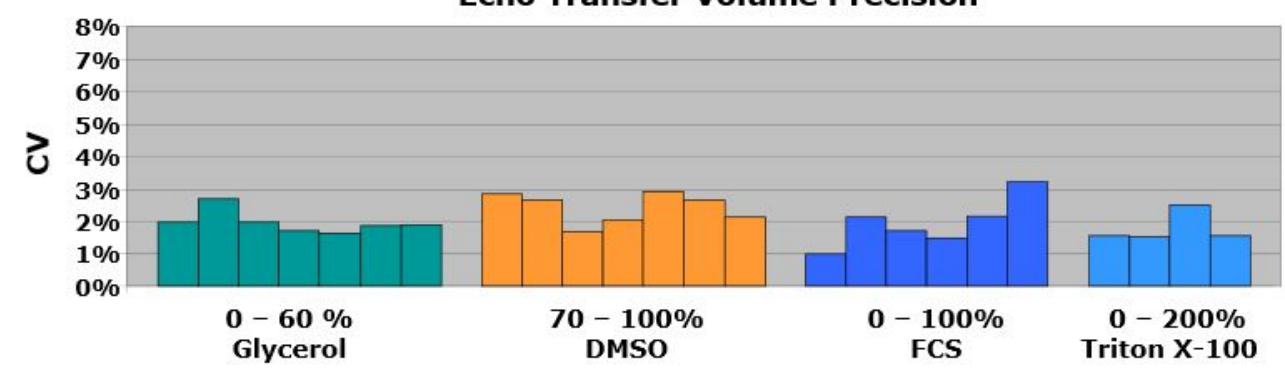


Fig. S2 Legend: Range of fluid viscosity and surface tension compatible with acoustic droplet ejection. Viscosity ranges from $0.39 \mathrm{cP}$ for $90: 10$ acetonitrile: $\mathrm{H}_{2} \mathrm{O}$, to $18 \mathrm{cP}$ for $30 \%$ PEG 3350 (not shown). Surface tension ranges from 21 dyne/cm for isopropyl alcohol to 72 dyne/cm for $1 X$ PBS: $\mathrm{H}_{2} \mathrm{O}$ (A). (B) ADE droplet transfer performance: absolute transfer volume and coefficient of variation is consistent for a wide range of sample fluids. The table shows the performance of acoustic fluid calibrations for four fluid classes: glycerol (GP, 0-60\%, 10\% steps), dimethyl sulfoxide (DMSO 70-100\%, 5\% steps), fetal calf serum (FCS) (BP, 0-100\%, 20\% steps), Triton $\mathrm{X}-100$ in water (SP, $0-200 \%$ of the critical micelle concentration (CMC), 0\%, 5\%, 14\%, 200\%). 
A

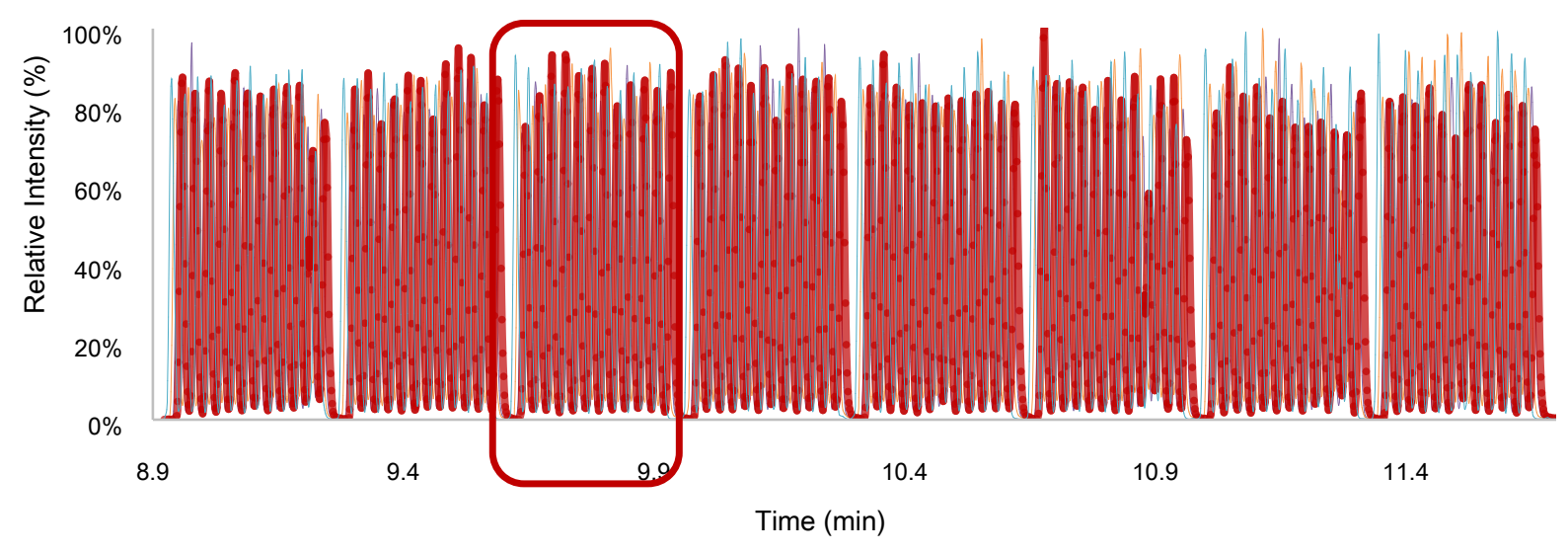

Omeprozole $\longrightarrow$ Midazolam $\longrightarrow$ Quinidine $\longrightarrow$ Bupropion

B

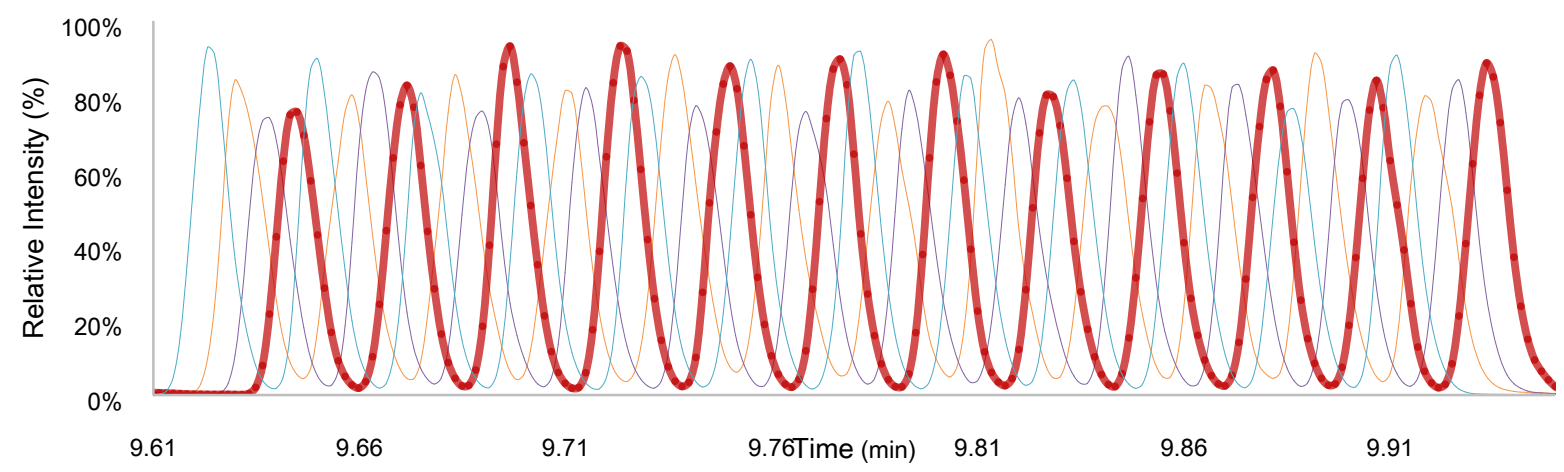

Fig. S3 Legend:

Multiplexed analysis with ADE-OPI-MS. (A) MS trace of 384 samples measured with multiplexing in $<170 \mathrm{sec}(2.2 \mathrm{~Hz})$ with four different compounds (omeprazole, quinidine, midazolam, bupropion) sequentially ejected from individual wells, $\mathrm{CV}$ in the range $3 \%-8 \%$. (B) One section of the 48 peaks shown in (A) enlarged. 
Figure S4: Sensitivity, carryover and linear dynamic range for the AEMS system

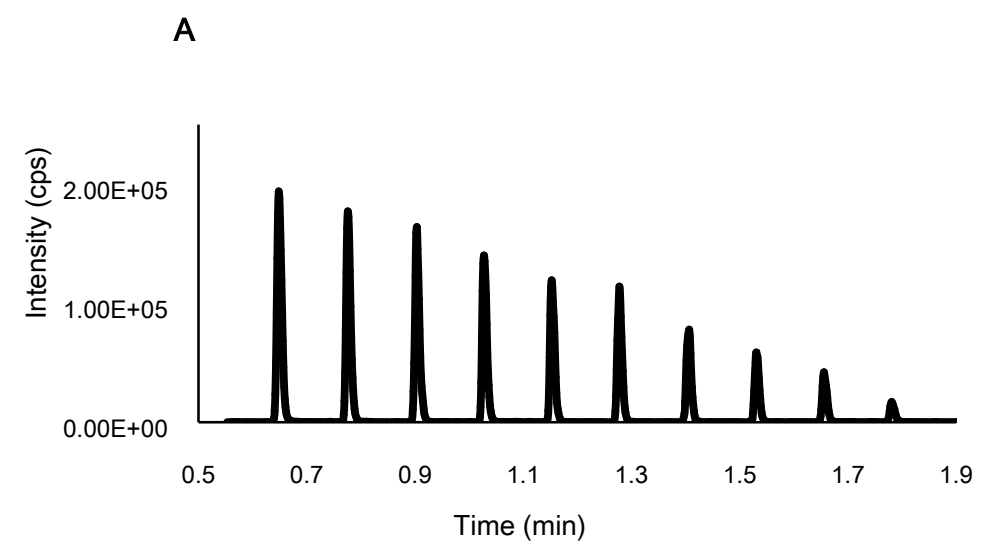

B

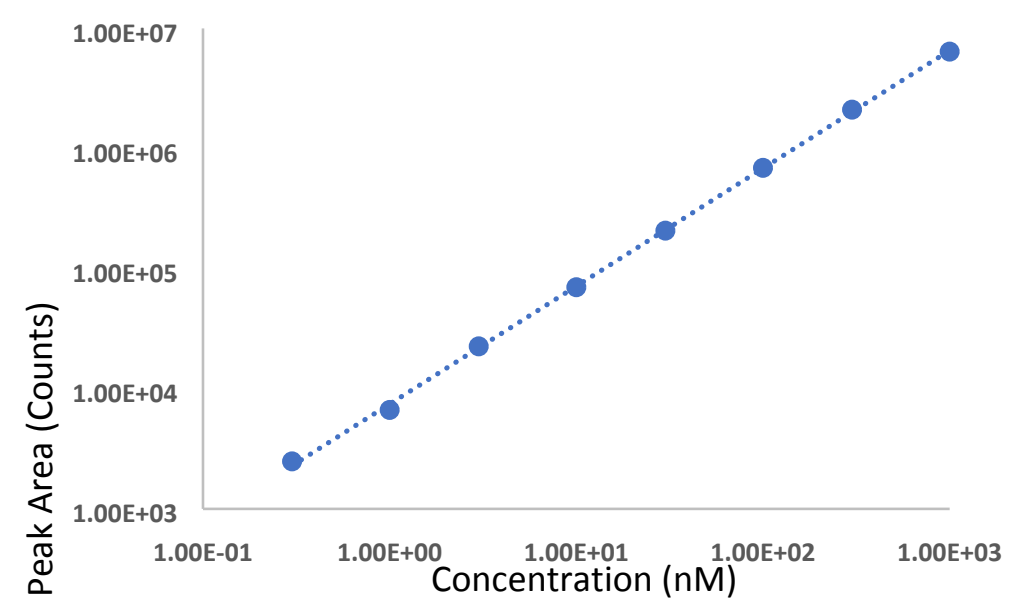

Fig. S4: Sensitivity, carryover and linear dynamic range for the AEMS system. (A) Analytical sensitivity was evaluated by injecting a droplet ladder (10 droplets to 1 droplet, $25 \mathrm{~nL}$ to $2.5 \mathrm{~nL}$ ) of $25 \mathrm{nM}$ propranolol. Sample droplets are interleaved with blank droplets, no carryover is observed. (B) Single sample drop (2.5 nL) analysis for dextromethorphan. Data performed with standard curves from $0.3 \mathrm{nM}$ to $1 \mu \mathrm{M}$. 
Figure S5: Matrix Tolerance Assessment with Detergent Samples

A

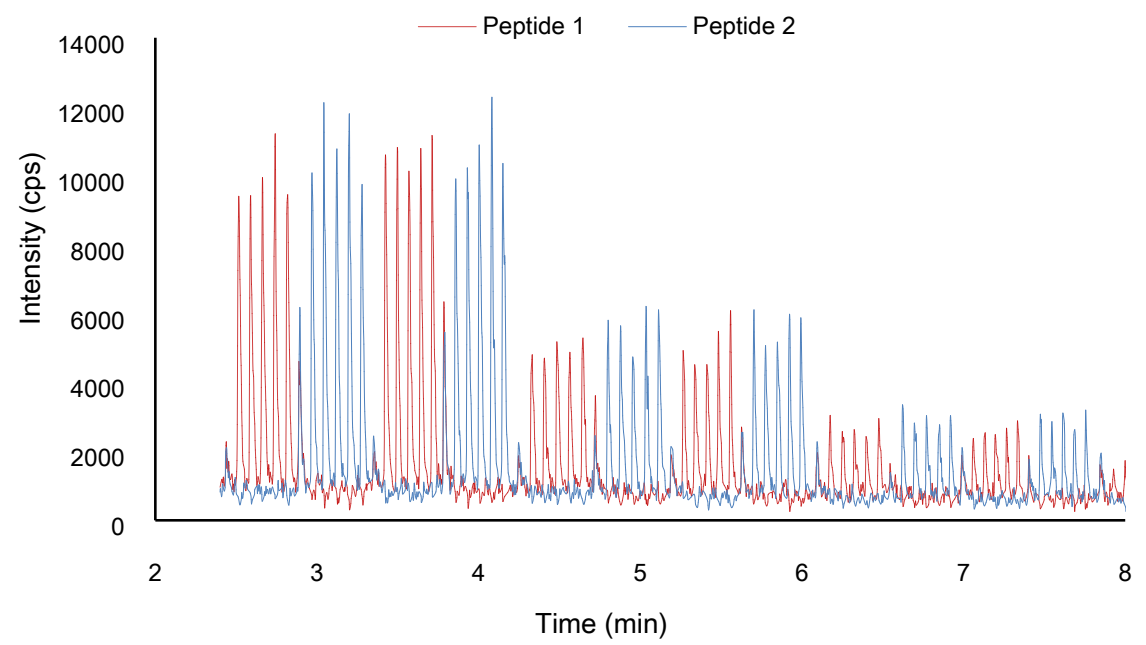

B

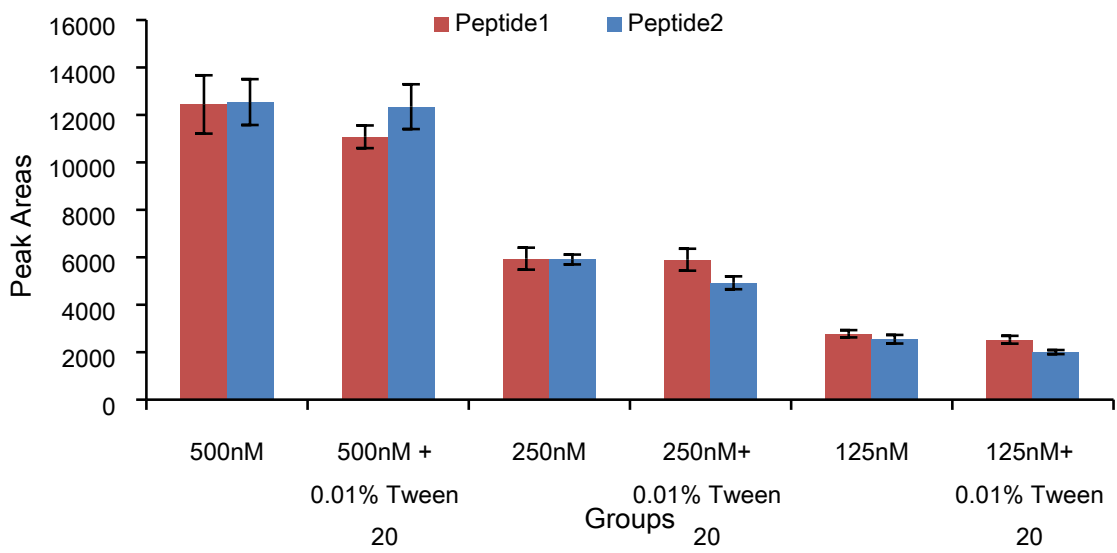




\section{Fig. S5 Legend:}

Matrix tolerance assessment with detergent samples. (A) MS traces of two alternating 17-aa peptides (shown in pink and blue respectively) at three different concentration levels: $500 \mathrm{nM}$, $250 \mathrm{nM}$, and $125 \mathrm{nM}$ (5 peaks of each). Additional peaks in between each group of 5-peaks represent signal from 1:1 mixture of both peptides at 100nM @2.4min, 3.4min, 4.3min, 5.3min, 6.2min, and 7.2min; 250nM@2.6min \&3.6min, 125nM@4.5min and 5.5min, and 72.5nM@6.5 and 7.4min, respectively. (B) Histogram showing the peak areas of two model peptides in buffer alone and with a commonly used detergent, 0.01\% Tween 20 . 
Figure S6: Analyte Coverage: Demonstration of Antibody Detection.

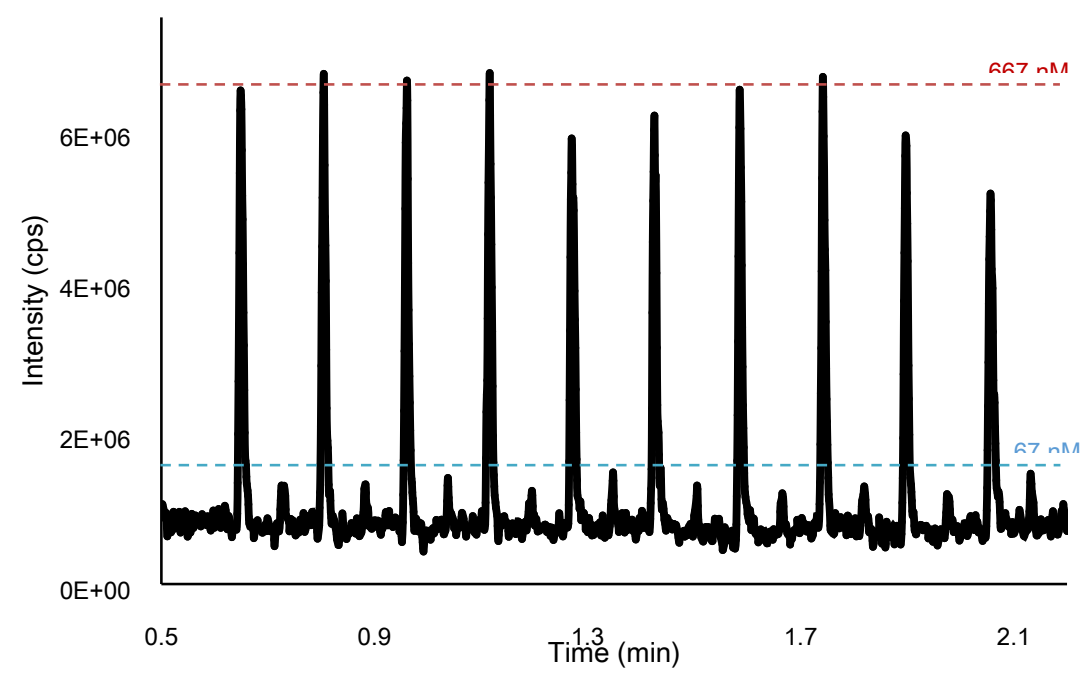

Fig. S6 Legend:

Analyte coverage: demonstration of antibody detection. ADE-OPI-MS analysis of antibody standard (MW 150 kDa) with alternating injections of $12.5 \mathrm{~nL}$ standards at a concentration of $667 \mathrm{nM}$ and $67 \mathrm{nM}$, respectively. The estimated LOD is $<1$ fmole loading. 


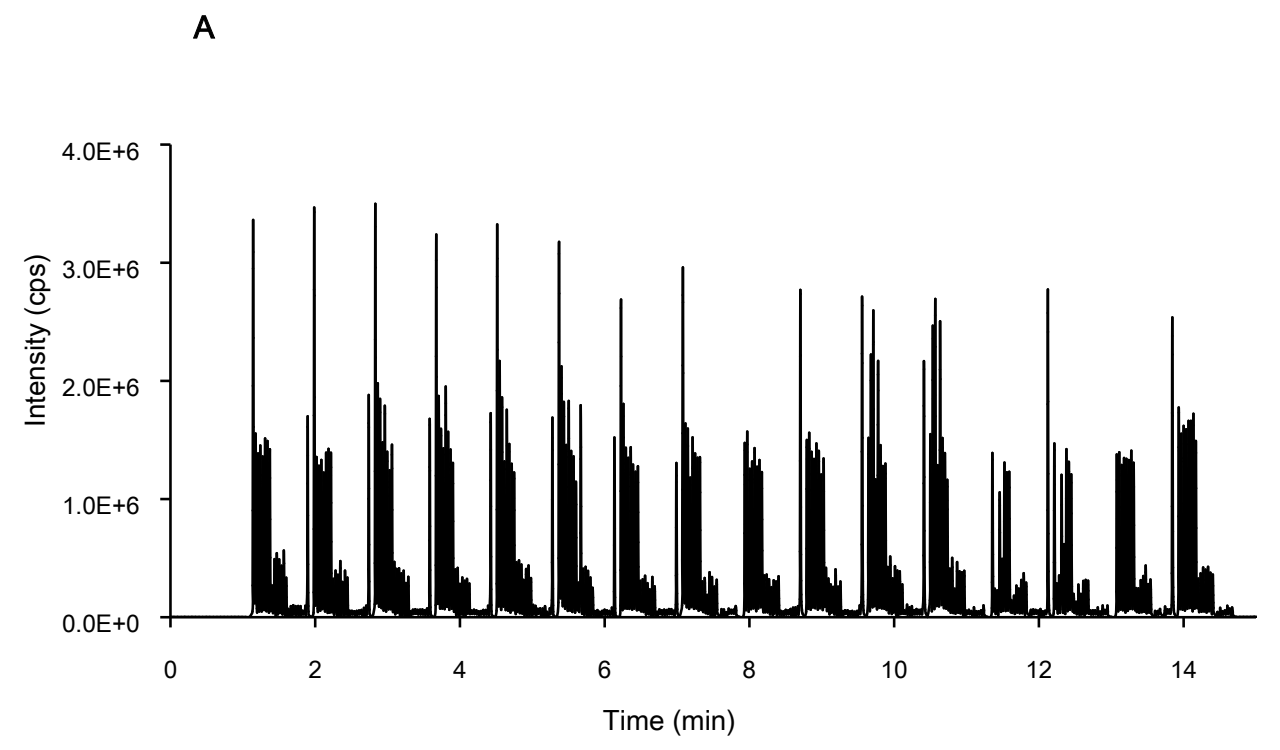

B

C
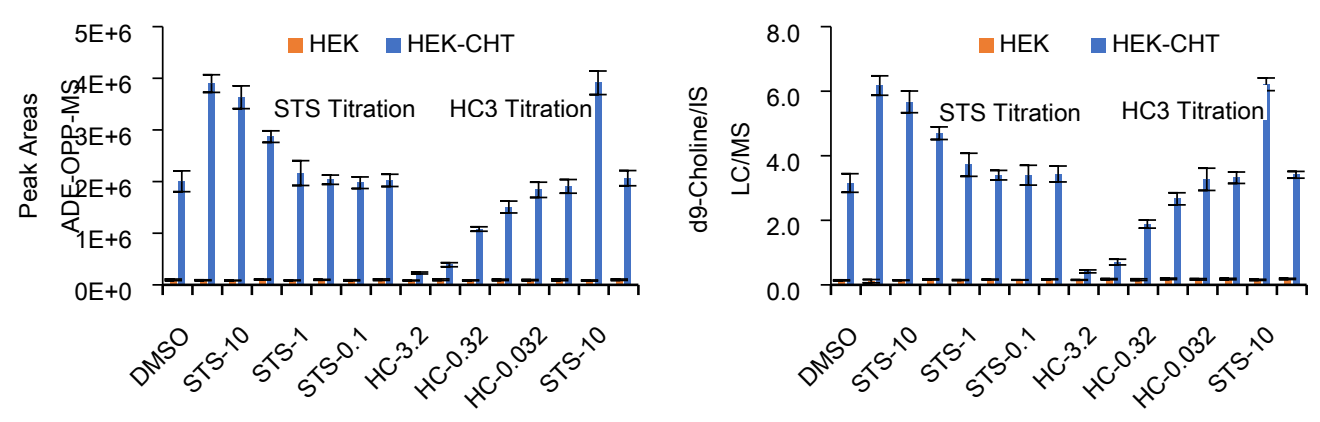

Fig. S7 Legend:

(A) ADE-OPI-MS experimental results for a full 384-well plate for the CHT uptake assay. D9labeled choline was monitored due to the high endogenous choline signal. All samples in the 384-wells were analyzed in $\sim 14$ min. Lysed cell samples were analyzed using the ADE-OPI-MS platform (B) and compared to a conventional LC-MS method (C) $)^{4}$. The data shown in (B) and 
(C) includes two modulators: hemicholinium-3 (HC), a known inhibitor and staurosporine (STS), a known activator that is dosed at a range of concentrations ${ }^{5}$. On the $\mathrm{x}$-axis the number after HCor STS- represent the dose concentrations (in $\mu \mathrm{M}$ ). HEK parental cell line was used as background in addition to the CHT overexpressed cell line (HEK-CHT). The ADE-OPI-MS platform increased the analysis speed 10 times and reduced sample volume 500 times while delivering equivalent data quality and concordance for both inhibition and activation profiles of the two modulators. 


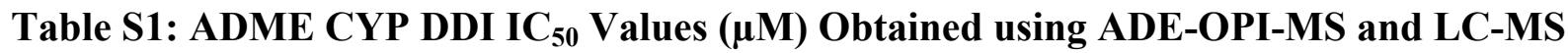

\begin{tabular}{|c|c|c|c|c|c|c|}
\hline \multirow[b]{2}{*}{ Compound } & \multicolumn{3}{|c|}{ ADE-OPI-MS } & \multicolumn{3}{|c|}{ LC/MS } \\
\hline & $1 \mathrm{~A} 2$ & 2D6 & 3A4 & $1 \mathrm{~A} 2$ & 2D6 & 3A4 \\
\hline $\mathrm{A}$ & 22.4 & 0.03 & $\geq 30.0$ & 13.5 & 0.03 & 9.2 \\
\hline $\mathrm{B}$ & $\geq 30.0$ & 0.25 & 0.69 & $\geq 30.0$ & 0.17 & 0.13 \\
\hline $\mathrm{C}$ & $\geq 30.0$ & $\geq 30.0$ & 0.10 & $\geq 30.0$ & $\geq 30.0$ & 0.07 \\
\hline $\mathrm{D}$ & $\geq 30.0$ & $\geq 30.0$ & 0.03 & $\geq 30.0$ & 26.1 & 0.03 \\
\hline $\mathrm{E}$ & 1.3 & 18.8 & 7.6 & 0.2 & 16.3 & 6.3 \\
\hline $\mathrm{F}$ & $\geq 30.0$ & 0.14 & 0.04 & $\geq 30.0$ & 0.09 & 0.07 \\
\hline $\mathrm{G}$ & $\geq 30.0$ & $\geq 30.0$ & $\geq 30.0$ & $\geq 30.0$ & $\geq 30.0$ & $\geq 30.0$ \\
\hline $\mathrm{H}$ & 1.0 & 7.6 & 0.12 & 1.2 & 6.2 & 0.12 \\
\hline I & $\geq 30.0$ & 20.7 & 7.4 & $\geq 30.0$ & 24.8 & 6.8 \\
\hline $\mathrm{J}$ & $\geq 30.0$ & $\geq 30.0$ & 0.08 & 14.9 & $\geq 30.0$ & 0.09 \\
\hline K & $\geq 30.0$ & 15.6 & 26.6 & $\geq 30.0$ & 17.4 & 16.3 \\
\hline $\mathrm{L}$ & $\geq 30.0$ & $\geq 30.0$ & 1.3 & 19.9 & $\geq 30.0$ & 1.6 \\
\hline $\mathrm{M}$ & 0.09 & 3.1 & 14.7 & 0.11 & 0.8 & 9.9 \\
\hline $\mathrm{N}$ & $\geq 30.0$ & 6.8 & $\geq 30.0$ & $\geq 30.0$ & 3.6 & $\geq 30.0$ \\
\hline $\mathrm{O}$ & 0.05 & 0.11 & 0.03 & 0.03 & 0.06 & 0.03 \\
\hline $\mathrm{P}$ & $\geq 30.0$ & $\geq 30.0$ & $\geq 30.0$ & $\geq 30.0$ & $\geq 30.0$ & $\geq 30.0$ \\
\hline
\end{tabular}

\section{Table S1 Legend:}

Calculated $\mathrm{IC}_{50}$ 's of 16 test compounds to three different CYP isoforms (1A2, 2D6, 3A4), with both ADE-OPI-MS and LC-MS platforms. 
Table S2: Z’ Metrics Determined for HT-Pharmacology Assay

\begin{tabular}{|l|c|c|c|}
\hline & $Z^{\prime}$ & IC50 for HC3 & EC50 for STS \\
\hline LC/MS without IS & 0.53 & $0.37 \mu \mathrm{M}$ & $3.8 \mu \mathrm{M}$ \\
\hline LC/MS with IS & 0.63 & $0.31 \mu \mathrm{M}$ & $3.9 \mu \mathrm{M}$ \\
\hline ADE-OPI-MS & 0.71 & $0.31 \mu \mathrm{M}$ & $2.4 \mu \mathrm{M}$ \\
\hline
\end{tabular}

\section{Table S2 Legend:}

Shown in the table are the Z' factors to assess the performance for 3 assays. For LC/MS, Z' $=$ 0.53 or 0.63 (without or with an IS). For ADE-OPI-MS, Z' $=0.71$ and there is no need for an IS. Calculation of Z' score for inhibition CHT assay: samples with DMSO were treated as ZPEs (negative control with $0 \%$ effect), and the highest dose of HC-3 samples were used as HPEs (positive control with 100\% effect). 
Movie S1: ADE-OPI-MS system in operation.

\section{Move S1 Legend:}

The movie shows an ADE-OPI-MS system in operation from a 384-well microplate. Video credit: Chang Liu, SCIEX. 
1. Dawes, T. D.; Turincio, R.; Jones, S. W.; Rodriguez, R. A.; Gadiagellan, D.; Thana, P.; Clark, K. R.; Gustafson, A. E.; Orren, L.; Liimatta, M., Compound transfer by acoustic droplet ejection promotes quality and efficiency in ultra-high-throughput screening campaigns. J. Lab. Autom. 2016, 21 (1), 64-75. 2. Hadimioglu, B.; Stearns, R.; Ellson, R., Moving liquids with sound: the physics of acoustic droplet ejection for robust laboratory automation in life sciences. J. Lab. Autom. 2016, 21 (1), 4-18. 3. $\quad$ Sackmann, E. K.; Majlof, L.; Hahn-Windgassen, A.; Eaton, B.; Bandzava, T.; Daulton, J.; Vandenbroucke, A.; Mock, M.; Stearns, R. G.; Hinkson, S., Technologies that enable accurate and precise nano-to milliliter-scale liquid dispensing of aqueous reagents using acoustic droplet ejection. $J$. Lab. Autom. 2016, 21 (1), 166-177.

4. Kapinos, B.; Liu, J.; Piotrowski, M.; Keefer, J.; Holder, B.; Janiszewski, J.; Zhang, H.; Troutman, M., Development of a high-performance, enterprise-level, multimode LC-MS/MS autosampler for drug discovery. Bioanalysis 2017, 9 (21), 1643-1654.

5. Choudhary, P.; Armstrong, E. J.; Jorgensen, C. C.; Piotrowski, M.; Barthmes, M.; Torella, R.; Johnston, S. E.; Maruyama, Y.; Janiszewski, J. S.; Storer, R. I., Discovery of compounds that positively modulate the high affinity choline transporter. Front. Mol. Neurosci. 2017, 10, 40. 